\title{
Pierre Robin Sequence: Diagnostic Difficulties Faced while Differentiating Isolated and Syndromic Forms
}

\author{
Girish Gulab Meshram ${ }^{1, *}$, Neeraj Kaur², Kanwaljeet Singh Hura ${ }^{3}$
}

\begin{abstract}
Pierre Robin sequence (PRS) is characterized by the triad of retrognathia, glossoptosis, and airway obstruction. PRS may occur in isolation or in conjunction with other syndromes. Distinguishing isolated and syndromic forms of PRS helps clinicians decide the management plan. We describe two cases of PRS of Indian ethnicity and describe some of the difficulties that we faced while distinguishing isolated PRS from syndromic PRS. Both cases had a similar clinical presentation. However, one of the cases had a positive family history of congenital deafness and cleft palate, whereas the other case had apparent upper limb anomalies. These facts heightened the suspicion of an associated syndrome. However, based on the available facts and after thorough investigations, a tentative diagnosis of isolated PRS was made for both the patients. Both the cases were managed conservatively and were advised a long-term follow-up. When the associated anomalies are few, minor or concealed at birth, longitudinal follow-up of all PRS cases combined with thorough diagnostics including chromosomal analysis could help differentiate syndromic PRS from isolated PRS. Regardless, all cases of PRS require a multidisciplinary approach.
\end{abstract}

\section{KEYWORDS}

Pierre Robin syndrome; retrognathia; amniotic band syndrome; limb deformities

\section{AUTHOR AFFILIATIONS}

${ }^{1}$ Department of Pharmacology, Atal Bihari Vajpayee Institute of Medical Sciences and Research and Dr. Ram Manohar Lohia Hospital, New Delhi, India

2 Department of Radiology, The University of Texas Health Science Centre, San Antonio, Texas, USA

${ }^{3}$ Department of Paediatrics, Richmond University Medical Centre, Staten Island, New York, USA

* Corresponding author: Department of Pharmacology, Atal Bihari Vajpayee Institute of Medical Sciences and Dr. Ram Manohar Lohia Hospital, New Delhi 110001, India; e-mail: drgirish23@yahoo.co.in

Received: 10 June 2019

Accepted: 7 February 2020

Published online: 10 August 2020

Acta Medica (Hradec Králové) 2020; 63(2): 86-90

https://doi.org/10.14712/18059694.2020.23

(c) 2020 The Authors. This is an open-access article distributed under the terms of the Creative Commons Attribution License (http://creativecommons.org/licenses/by/4.0), which permits unrestricted use, distribution, and reproduction in any medium, provided the original author and source are credited. 


\section{INTRODUCTION}

Pierre Robin sequence (PRS) is the triad of retrognathia, glossoptosis, and airway obstruction. Cleft palate is frequently encountered, but is not considered as a prerequisite for the diagnosis (1). Epidemiological data are sparse, though available evidence suggests the incidence of PRS ranges from 1 in 5600 to 1 in 14000 live births $(1,2)$. Symptoms include varying degrees of upper airway obstruction and feeding problems. PRS occurs in isolation or as a part of other syndromes such as Stickler, velocardiofacial, and Treacher-Collins syndromes (3). Because the severity of symptoms, presence of long-term sequalae, requirement of surgery, and mortality rate in syndromic PRS are much higher than non-syndromic/isolated PRS, early differentiation between the two is beneficial to clinicians as it increases their preparedness and helps them take decisions regarding the management plan $(1,4,5)$. However, the phenotypic heterogenicity of the various associated syndromes, make it very hard for clinicians to distinguish between the two (3). This ordeal is more pronounced when the associated congenital anomalies are few, minor, or concealed at birth as in the case of Stickler syndrome (6). The lack of chromosomal diagnostics in developing nations for phenotype-genotype correlation adds to this obstacle (7). In this paper, we describe two cases of PRS of Indian ethnicity and describe some of the difficulties that we faced while differentiating syndromic PRS from non-syndromic PRS. This paper also includes the review of up to date literature and the latest trends in management of PRS.

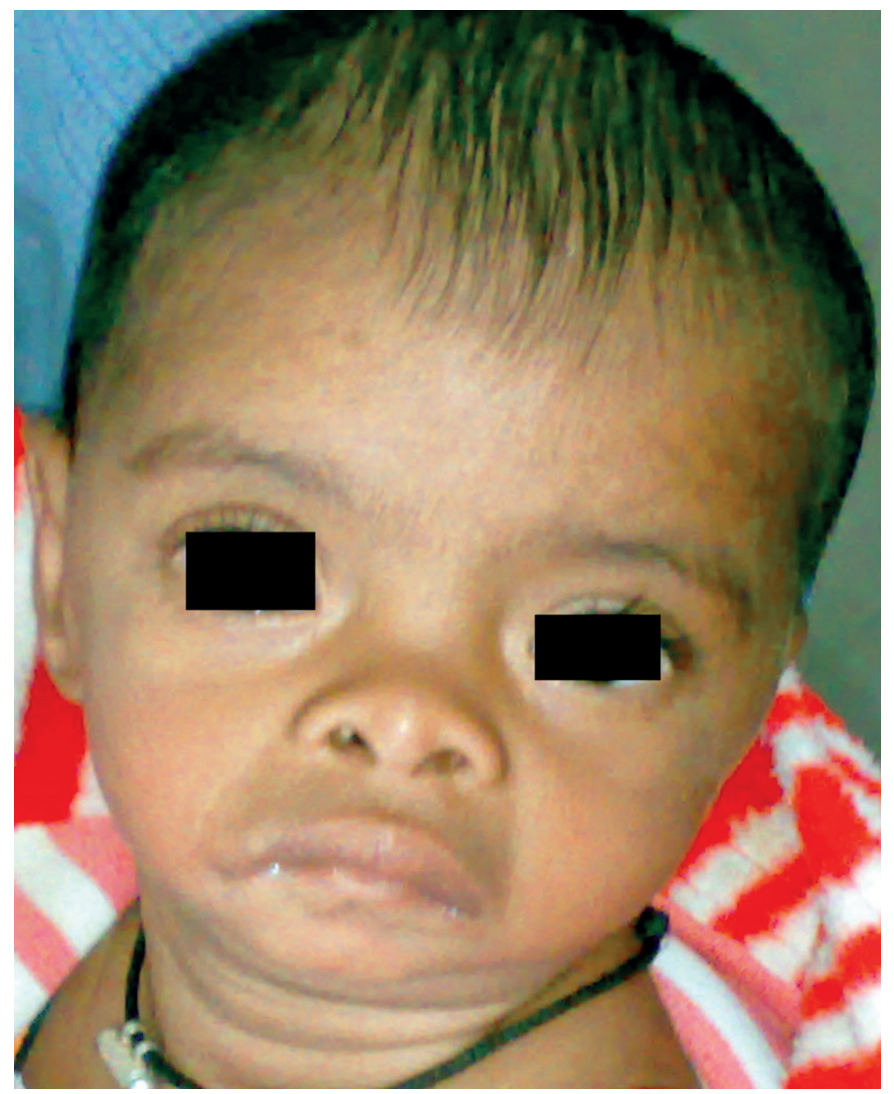

Fig. 1 A 2-month-old infant with isolated Pierre Robin sequence (Case 1)

\section{CASE PRESENTATION}

Case 1 was a 2-month-old male infant and Case 2 was a 2-day-old male newborn (Figure 1 and Figure 2). Both were of Indian ethnicity and had visited the pediatric outpatient department with their parents. Both cases were referred to the department of pediatrics from peripheral healthcare centers, where specialists were not available.

Both patients presented with congenital facial deformities and difficulty in breathing. Case 1 had difficulty in breathing while lying down, whereas Case 2 had respiratory distress in both lying down and upright positions. Case 1 was born via normal vaginal delivery at 37 weeks of gestation with a birth weight of $3 \mathrm{~kg}$ (appropriate for gestational age) without complications and was second in birth order. Case 2 was born to a primigravida via normal vaginal delivery at 40 weeks of gestation with a birth weight of $3.6 \mathrm{~kg}$ (appropriate for gestational age), but the pregnancy was complicated with preeclampsia, which was managed with magnesium sulfate. The parents of both patients had a non-consanguineous marriage. Case 1 had a cousin with history of cleft palate and congenital deafness, whereas none of the close family members of Case 2 had a history of congenital anomalies. History of alcohol consumption during pregnancy or radiation exposure was absent in mothers of both patients. Except for consumption of magnesium sulfate by the mother of Case 2, no significant drug history was present in both patients.

On examination, both patients had small-sized mandibles, which were deflecting backwards (micrognathia) (Figure 1 and Figure 2). Cleft palate and bifid uvula were absent in both cases. Examination of the cardiovascular, musculoskeletal, and central nervous systems of Case $1 \mathrm{did}$ not indicate the presence of any other congenital anomaly. Examination of the cardiovascular and central nervous system of Case 2 did not reveal any abnormality. However, examination of the musculoskeletal system of Case 2 revealed absence of four digits in the right upper limb (Figure 2). The respiratory rates of Case 1 and Case 2 were 42 and 48 breaths/minute, respectively. Both cases had

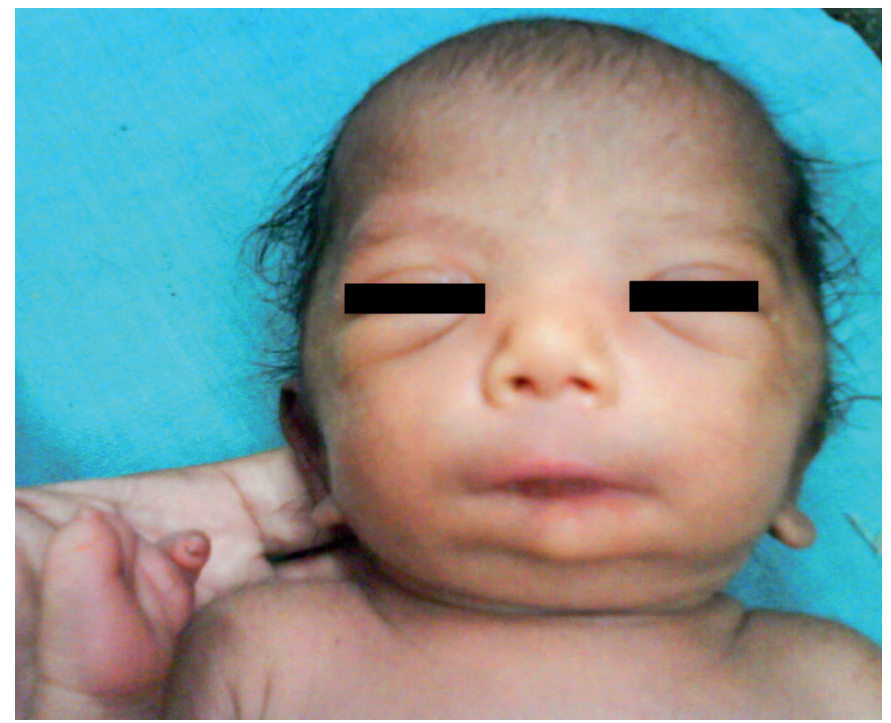

Fig. 2 A 2-day-old neonate with Pierre Robin sequence and absence of 4 digits in the right upper limb (Case 2). 
labored breathing without any signs of cyanosis. Case 2, in addition, had mild intercostal recessions. The complete blood count (red blood cell count, hemoglobin, hematocrit, white blood cell count, and platelet count), liver function tests (total bilirubin, serum aminotransferase, and serum alkaline phosphatase), and kidney function tests (blood urea nitrogen, and serum creatinine) levels in both patients were within reference ranges. X-ray chest of both patients did not reveal any abnormality. X-ray of the right upper limb of Case 2 showed absence of the phalanges in four digits, except the thumb. The metacarpals and carpals of all five digits appeared normal. Two-dimensional echocardiography findings of both patients were found to be normal. Ophthalmological examination of both patients did not show any abnormalities. Otoscopy and otoacoustic emission screening test for sensorineural deafness were negative for both patients. The background of congenital deafness in one of the family members of Case 1 made him a high-risk patient for hearing loss. Hence, we wanted to thoroughly investigate him further to rule out the same. Hence, we advised him an auditory brainstem response test (ABR). ABR is a noninvasive measure of sensorineural and conductive hearing loss, wherein electrodes are attached to the scalp of the child via stickers and the responses of the auditory nerve, cochlea and brainstem to various auditory stimuli are measured electronically. In order to assist the diagnosis, rule out associated syndromes and determine the hereditary pattern, we advised genetic testing to both patients. For Case 1 we advised detection of deletion of 22q11 chromosome via fluorescence in situ hybridization to rule out association with velocardiofacial syndrome, and mutations in COL2A1 gene via polymerase chain reaction to rule out association with Stickler syndrome. For Case 2 we advised detection of mutations in SF3B4 and TCOF1 genes via polymerase chain reaction to rule out an association with acrofacial dysostosis syndrome and Treacher-Collins syndrome, respectively.

Both ABR and molecular tests were declined by the patient's families due to their financial limitations.

As Case 1 had a family history of congenital hearing loss and the presence of sensorineural hearing loss was not completely ruled out due to the impending ABR, we considered PRS associated with Stickler syndrome or velocardiofacial syndrome in our differential diagnosis. However, as no other apparent malformations were present in Case 1 , suggestive of these syndromes, we made a diagnosis of isolated PRS. Similarly, due to the presence of upper limb anomalies in Case 2, we initially considered PRS associated with acrofacial dysostosis syndrome or Treacher-Collins (Rodriguez, Nager, and Miller subtypes) syndrome. However, the limb anomalies in Case 2 could be also explained by the presence of a constricting amniotic band in the in utero period leading to autoamputation of the digits, which seemed more consistent. Hence, Case 2 too was diagnosed as a case of isolated PRS complicated due to an amniotic band.

Both patients were admitted and were advised prone positioning. Oxygen saturation levels of Case 1 and Case 2 were $98.2 \%$ and $93.4 \%$, respectively, at the time of admission. The breathing of Case 1 improved with prone-positioning. Hence, his parents were provided appropriate guidance of prone positioning the child when the respiratory distress increased. The breathing difficulty of Case 2 did not improve with prone positioning. Hence, a nasopharyngeal airway was placed and supplemental oxygen was provided, which improved the oxygen saturation to $98 \%$. Both respiratory and feeding difficulties of Case 2 subsided on day- 5 of admission. Both patients were subsequently discharged and anticipatory guidance was provided to their parents. A longitudinal follow-up was advised for both patients with consultation with various faculties such as pediatrics, ophthalmology, otorhinolaryngology, orthopedics, and dentistry. Both patients were requested to follow-up at regular intervals of 2 months for a minimum period of 5 years. Both the cases have not followed up so far. Before their discharge, a written informed consent was obtained from the legally authorized representatives of both patients for anonymized patient information to be published in this case report.

\section{DISCUSSION}

PRS is a set of abnormalities affecting the head and face, consisting of micrognathia (small and symmetrically receded mandible), glossoptosis (tongue that obstructs the posterior pharyngeal space), and resultant airway obstruction (3). Cleft palate is found in almost $90 \%$ of the cases (8). However, in both our cases cleft palate was absent.

Ultrasound imaging helps in the prenatal diagnosis of severe cases of PRS and helps specialists plan the further course of pregnancy and postnatal care $(8,9)$. As both our cases had been evaluated outside our hospital during their prenatal period, we could not detect the presence of abnormal ultrasound findings, suggestive of PRS, such as retrognathia/micrognathia. Also, maternal factors such as oligohydramnios, multigravida pregnancy, and uterine anomalies which could hinder the mandibular growth of the child could be detected early via ultrasonography (9).

The various hypotheses suggested behind the etiology of PRS include in utero mechanical compression of the mandible, delay in neurological maturation of the nerves supplying the affected areas, and dysregulation of the rhombocephalus $(2,3,10)$. De novo mutations in the SOX9 and KCNJ2 genes are linked with non-syndromic/isolated PRS $(2,10)$. Isolated PRS accounts for around $20-40 \%$ of all cases of PRS (2). Once afflicted with isolated PRS, the inheritance pattern followed is autosomal dominant (9). Common medical syndromes with which PRS occurs in conjunction include Stickler, Nager, velocardiofacial, 22q11 deletion, fetal alcohol, and Treacher-Collins syndromes $(11,12)$. Genetic mutations such as COL2A1, SF3B4, and TCOF1 causing these associated syndromes are implicated in the etiology of syndromic PRS $(10,11)$. Once afflicted with syndromic PRS, the inheritance pattern followed is the same as the associated condition $(9,11)$. In both our cases, we could not determine the genetic basis of the etiology via chromosomal studies due the economic constraints of our patients. Differentiating isolated PRS from syndromic PRS provides insights into the etiology, which in turn determines the pathogenesis and the clinical presentation (4). 
In the outpatient department, patients with PRS usually present with a characteristic bird-like face with variable degrees of breathing/feeding difficulties, as found in both our patients $(2,13)$. When the triad of PRS is spotted, the physician should have an heightened index of suspicion for other anomalies and a detailed family history of congenital anomalies should be elucidated $(6,8)$. As one of our cases had a family history of congenital hearing loss and cleft palate in a second-degree relative, and the other had apparent upper limb malformations, we initially suspected syndromic PRS in both the cases. Cleft palate and sensorineural hearing loss along with PRS is commonly associated with Stickler and velocardiofacial syndrome (14). Similarly, presence of limb malformations along with PRS is commonly associated with acrofacial dysostosis or Treacher-Collins (Rodriguez, Nager, and Miller subtypes) syndromes $(9,14)$.

As more than 50 syndromes have been associated with PRS and each of these syndromes have a vast heterogenicity in their respective presentations, it often becomes very difficult for clinicians to accurately identify the associated syndrome $(3,5)$. We faced a similar predicament. Also, when the anomalies are not apparent at birth or are minor, as in the case of Stickler's syndrome, diagnosis of syndromic PRS is often missed $(2,6)$. In such situations, a longitudinal follow-up with detailed chromosomal diagnostics has been suggested for identification of the associated syndrome $(4,8)$. However, in most economically-deprived nations, genotype-phenotype correlation is not commonly carried out due to economic constraints of patients, or unavailability of expert knowledge (7). Such was our case too.

In each of our cases, via radiological imaging and other diagnostics, we systematically tried to rule out other anomalies. However, due to the absence of other detectable congenital anomalies in Case 1 and the upper limb deformities in Case 2 explainable by an in utero amniotic band, we diagnosed both cases as isolated PRS $(2,15)$. However, both these diagnoses were formed without the support of genetic studies. It has been reported that if PRS infants are followed-up until childhood along with appropriate genetic diagnostics, the diagnosis changes in $25 \%$ of the cases because with course of time the symptomology of numerous syndromes becomes more apparent or new anomalies are developed $(4,8)$.

Many believe that delineating syndromic PRS from isolated PRS is crucial as it influences the choice and outcome/ efficacy of the management strategy (4). Clinicians mainly focus on the management of the morbidities caused due to PRS $(1,2)$. The severity of the airway obstruction even in non-syndromic PRS cases requires the use of fiberoptic investigations for exclusion of congenital abnormalities as well as for local description of the degree of obstruction (16). Managing respiratory obstruction is prioritized over addressing feeding dysfunction $(2,3)$. Algorithms such as the Vancouver Classification for the airway management of PRS have been devised by institutes to individualize the selection of the management plan (5). However, no consensus is present regarding the selection of the management plan in the literature (12). Prone positioning, placing a nasopharyngeal airway, providing continuous positive airway pressure, and using a laryngeal mask airway are the common noninvasive techniques used to relieve the airway obstruction (1-3). Supplemental feeding using a nasogastric/orogastric tube (mild cases) and gastronomy tube (chronic and persistent cases) helps resolving feeding difficulties $(1,13)$. Almost $70 \%$ of PRS cases respond to conservative management, as our patient's did (4).

As a substantial set of the population with PRS achieve normal or near-normal mandibular size within a few years of birth, the cornerstone in the management of PRS is conservative therapy $(2,4)$. Hence, we chose to manage both the patients conservatively and follow them up on regular intervals. Surgical interventions are only needed when all conservative measures are exhausted $(3,4)$. A report suggested that syndromic PRS cases have a higher requirement of surgical interventions and have poorer outcomes, as compared to isolated PRS (5). Surgical therapies used to relieve airway obstruction include tongue-lip adhesion, mandibular distraction osteogenesis, subperiosteal release of the floor of the mouth, and tracheostomy $(3,8)$.

It has been observed that the mortality and complication rates in syndromic PRS are much higher than isolated PRS $(1,17)$. The secondary effects of PRS include failure to thrive, developmental delays, dental anomalies, gastroesophageal reflux, sleeping difficulties, speech disorders, psychological disorders, cardiac failure, and brain damage $(1,3)$. Hence, considering the constellation of sequelae and complications, which could arise in the clinical course of the disease, a long-term follow-up by a multidisciplinary team of experts is essential for developing an individualized management strategy for patients with PRS $(2,3,8)$.

\section{CONCLUSIONS}

We presented two cases of isolated PRS of Indian ethnicity. We faced numerous hurdles while differentiating isolated PRS from syndromic PRS, which we have enumerated in our case report. We managed both patients successfully via symptomatic conservative management. Differentiating isolated PRS from syndromic PRS helps clinicians take decisions regarding the management plan, taking into consideration the differences in their complication, morbidity, and mortality rates. Regardless, we recommend a longitudinal follow-up of all PRS cases via a multidisciplinary team, augmented with genetic diagnostics, for devising a dynamic and personalized management plan.

\section{CONFLICT OF INTEREST}

The authors disclose no conflicts of interest.

\section{REFERENCES}

1. Logjes RJH, Haasnoot M, Lemmers PMA, et al. Mortality in Robin sequence: identification of risk factors. Eur J Pediatr 2018; 177: 781-9.

2. Gangopadhyay N, Mendonca DA, Woo AS. Pierre Robin Sequence. Semin Plast Surg 2012; 26: 76-82.

3. Evans KN, Sie KC, Hopper RA, Glass RP, Hing AV, Cunningham ML. Robin sequence: from diagnosis to development of an effective management plan. Pediatrics 2011; 127: 936-48.

4. Basart H, Paes EC, Maas SM, et al. Etiology and pathogenesis of robin sequence in a large Dutch cohort. Am J Med Genet A 2015; 167A: 1983-92. 
5. Li WY, Poon A, Courtemanche D, et al. Airway management in Pierre Robin sequence: the Vancouver classification. Past Surg (Oakv) 2017; 25: 14-20.

6. Vatlach S, Maas C, Poets CF. Birth prevalence and initial treatment of Robin sequence in Germany: a prospective epidemiologic study. Orphanet J Rare Dis 2014; 9: 9.

7. Ratan ZA, Zaman SB, Mehta V, Haidere MF, Runa NJ, Akter N. Application of fluorescence in situ hybridization (FISH) technique for the detection of genetic aberration in medical science. Cureus 2017; 9: e1325.

8. Cohen SM, Greathouse ST, Rabbani CC, et al. Robin sequence: what the multidisciplinary approach can do. J Multidiscp Healthc 2017; 10: 121-32.

9. Paladini D. Fetal micrognathia: almost always an ominous finding. Untrasound Obstet Gynecol 2010; 35: 377-84.

10. Xu JX, Kilpatrick N, Baker NL, Penington A, Farlie PG, Tan TY. Clinical and molecular characteristics of children with Pierre Robin sequence. Mol Syndromol 2016; 7: 322-8.

11. Yang Y, Yuan J, Yao X, et al. BMPR1B mutation causes Pierre Robin sequence. Oncotarget 2017; 8: 25864-71.
12. Muller-Hagedorn S, Buchenau W, Arand J, Bacher M, Poets CF. Treatment of infants with syndromic Robin sequence with modified palatal plates: a minimally invasive treatment option. Head Face Med 2017; 13: 4.

13. Paes EC, de Vries IAC, Penris WM, et al. Growth and prevalence of feeding difficulties in children with Robin sequence: a retrospective cohort study. Clin Oral Investig 2017; 21: 2063-76.

14. Bartzela TN, Carels C, Maltha JC. Update of 13 syndromes affecting craniofacial and dental structures. Front Physio 2017; 8: 1038.

15. Lopez-Munoz E, Becerra-Solano LE. An update on amniotic bands sequence. Arch Argent Pediatr 2018; 116: e409-e420.

16. Koukouvitaki M, Arkaoumanis PT, Brucknerova J, Brucknerova I. Clinical attitude to the patient with non-syndromic Pierre Robin Sequence with the cleft of soft palate and uvula - The necessity of fibroscopic investigation. Neuro Endocrinol Lett 2019; 40: 5-9.

17. Butow K, Morkel JA, Naidoo S, Zwahlen RA. Pierre Robin sequence: subdivision, data, theories, and treatment - Part 1: syndromic and nonsyndromic Pierre Robin sequence. Ann Maxillofac Surg 2016; 6: 35-7. 